\title{
ESTUDO FLUIDODINÂMICO DO ESCOAMENTO E ANÁLISE DA QUEDA DE PRESSÃO DE FLUIDOS NÃO-NEWTONIANOS EM SEÇÕES ANULARES
}

\author{
I. C. BICALHO, J. L. MOGNON, D. B. DOS SANTOS, C. H. ATAÍDE e C. R. DUARTE \\ Universidade Federal de Uberlândia, Departamento de Engenharia Química \\ E-mail para contato: chataide@ufu.br
}

\begin{abstract}
RESUMO - Durante a operação de perfuração, um fluido é bombeado através da coluna até o fundo do poço e retorna à superfície pelo espaço anular formado entre a coluna e a parede do poço. Este fluido deve atender requisitos que estão diretamente relacionados ao custo efetivo das operações de perfuração e também a segurança, sendo fundamental a correta previsão de seu escoamento. Desde que os custos anuais das operações de perfuração situam-se acima de bilhões de dólares, a indústria petrolífera tem investido pesado na pesquisa desse escoamento, sendo fundamental prever o comportamento do fluxo no espaço anular, a perda de carga ao longo do poço, a velocidade e eficiência no transporte dos cascalhos a fim de se otimizar a operação. Desta forma, buscou-se neste trabalho avaliar o escoamento laminar de fluidos não-Newtonianos em seções horizontais concêntricas/excêntricas com obstrução parcial, o que permitiu prever aspectos de campo de fluxo, incluindo perfis de velocidade e quedas de pressão.
\end{abstract}

\section{INTRODUÇÃO}

A compreensão dos fenômenos presentes no deslocamento de fluidos em espaços anulares é de considerável importância em diversas operações industriais, sendo foco de estudos nas indústrias químicas, alimentícias e petrolíferas, com destaque para as atividades de perfuração e completação de poços de petróleo (ESCUDIER et al., 2002). Como os custos das operações de perfuração são elevados, o investimento em pesquisas é algo muito vantajoso, pois melhoramentos desenvolvidos nessa atividade podem ser convertidos em altos lucros. Além disso, com a descoberta de novas tecnologias pode-se aumentar a produção com menor agressão ao meio ambiente (PEREIRA, 2006). Durante a atividade de perfuração de um poço, um fluido de perfuração é utilizado ao longo de toda a operação. Esse fluido é bombeado a partir da superfície por dentro da coluna de perfuração, saindo por orifícios na broca e chegando ao espaço anular formado entre a coluna e a parede do poço por onde ocorre o escoamento de retorno até a superfície. Utilizam-se vários tipos de fluidos de perfuração, sendo na sua maioria fluidos de comportamento não-Newtoniano (THOMAS, 2001). O estudo do escoamento na região anular é muito importante, uma vez que a ineficiência na remoção dos cascalhos de dentro do poço pode dificultar o processo de perfuração diminuindo a taxa de penetração e vida útil da broca, provocando perda de circulação, obstrução do anular e até mesmo interrompendo o processo, já que pode ocorrer prisão da coluna de perfuração. 
Para melhor entender a capacidade de carreamento do fluido de perfuração, deve-se compreender o escoamento existente na região anular entre a parede do poço e a coluna giratória. Tal escoamento depende de algumas variáveis tais como: o fluido de perfuração utilizado, a geometria do poço, a rotação da coluna, a vazão axial imposta, a concentração e dimensão dos cascalhos (LOUREIRO, 2004). Em particular, uma dificuldade que pode ocorrer no caso de poços inclinados ou horizontais é o acúmulo de material sólido na parte inferior do poço devido ao efeito gravitacional, formando um leito de cascalhos sedimentado (obstrução parcial). A presença de sólidos acumulados nessa região pode provocar desde um aumento no torque de acionamento da broca até a critica situação de entupimento do poço. Por esta razão é importante estudar os padrões de escoamento em espaços anulares parcialmente obstruídos e determinar as condições que garantam a eficiência de carreamento. Assim, o objetivo deste trabalho foi estudar com o auxílio de técnicas de Fluidodinâmica Computacional (CFD), o efeito de algumas variáveis, tais como, vazão de fluido, concentração de goma Xantana $(\mathrm{GX})$, excentricidade, rotação do tubo interno e presença de obstrução no anular, sobre os perfis de velocidade e queda de pressão no escoamento de fluidos nãoNewtonianos em canais anulares.

\section{MATERIAL E MÉTODOS}

O procedimento para a simulação numérica foi implementado empregando-se códigos comerciais de CFD. Para a construção da malha utilizou-se o software GAMBIT $^{\circledR} 2.3 .16$ e os cálculos numéricos foram conduzidos no software FLUENT ${ }^{\circledR} 14.0$.

\subsection{Equações Governantes}

Para um escoamento isotérmico, incompressível e em regime laminar de um fluido nãoNewtoniano em um anular, a modelagem matemática pode ser realizada com base nas equações de conservação da massa (Equação 1) e do balanço da quantidade de movimento (Equações 2, 3 e 4).

$$
\begin{aligned}
& \frac{1}{\mathrm{r}} \frac{\partial\left(\mathrm{rv}_{\mathrm{r}}\right)}{\partial \mathrm{r}}+\frac{1}{\mathrm{r}} \frac{\partial\left(\mathrm{v}_{\theta}\right)}{\partial \theta}+\frac{\partial\left(\mathrm{v}_{\mathrm{z}}\right)}{\partial \mathrm{z}}=0 \\
& \rho\left(\frac{\partial\left(\mathrm{v}_{\mathrm{r}}\right)}{\partial \mathrm{t}}+\mathrm{v}_{\mathrm{r}} \frac{\partial\left(\mathrm{v}_{\mathrm{r}}\right)}{\partial \mathrm{r}}+\frac{\mathrm{v}_{\theta}}{\mathrm{r}} \frac{\partial\left(\mathrm{v}_{\mathrm{r}}\right)}{\partial \theta}+\mathrm{v}_{\mathrm{z}} \frac{\partial\left(\mathrm{v}_{\mathrm{r}}\right)}{\partial \mathrm{z}}\right)=-\frac{\partial \mathrm{P}}{\partial \mathrm{r}}+\left(\frac{1}{\mathrm{r}} \frac{\partial\left(\mathrm{r} \tau_{\mathrm{rr}}\right)}{\partial \mathrm{r}}+\frac{1}{\mathrm{r}} \frac{\partial\left(\tau_{\mathrm{r} \theta}\right)}{\partial \theta}+\frac{\partial\left(\tau_{\mathrm{rz}}\right)}{\partial \mathrm{z}}+\frac{\tau_{\theta \theta}}{\mathrm{r}}\right)+\rho \mathrm{g}_{\mathrm{r}}(2) \\
& \rho\left(\frac{\partial\left(\mathrm{v}_{\theta}\right)}{\partial \mathrm{t}}+\mathrm{v}_{\mathrm{r}} \frac{\partial\left(\mathrm{v}_{\theta}\right)}{\partial \mathrm{r}}+\frac{\mathrm{v}_{\theta}}{\mathrm{r}} \frac{\partial\left(\mathrm{v}_{\theta}\right)}{\partial \theta}+\mathrm{v}_{\mathrm{z}} \frac{\partial\left(\mathrm{v}_{\theta}\right)}{\partial \mathrm{z}}+\frac{\mathrm{v}_{\mathrm{r}} \mathrm{v}_{\theta}}{\mathrm{r}}\right)=-\frac{1}{\mathrm{r}} \frac{\partial \mathrm{P}}{\partial \theta}+\left(\frac{1}{\mathrm{r}^{2}} \frac{\partial\left(\mathrm{r}^{2} \tau_{\mathrm{r} \theta}\right)}{\partial \mathrm{r}}+\frac{1}{\mathrm{r}} \frac{\partial\left(\tau_{\theta \theta}\right)}{\partial \theta}+\frac{\partial\left(\tau_{\theta \mathrm{z}}\right)}{\partial \mathrm{z}}\right)+\rho \mathrm{g}_{\theta}(3) \\
& \rho\left(\frac{\partial\left(\mathrm{v}_{\mathrm{z}}\right)}{\partial \mathrm{t}}+\mathrm{v}_{\mathrm{r}} \frac{\partial\left(\mathrm{v}_{\mathrm{z}}\right)}{\partial \mathrm{r}}+\frac{\mathrm{v}_{\theta}}{\mathrm{r}} \frac{\partial\left(\mathrm{v}_{\mathrm{z}}\right)}{\partial \theta}+\mathrm{v}_{\mathrm{z}} \frac{\partial\left(\mathrm{v}_{\mathrm{z}}\right)}{\partial \mathrm{z}}\right)=-\frac{\partial \mathrm{P}}{\partial \mathrm{z}}+\left(\frac{1}{\mathrm{r}} \frac{\partial\left(\mathrm{r} \tau_{\mathrm{rz}}\right)}{\partial \mathrm{r}}+\frac{1}{\mathrm{r}} \frac{\partial\left(\tau_{\theta \mathrm{z}}\right)}{\partial \theta}+\frac{\partial\left(\tau_{\mathrm{zz}}\right)}{\partial \mathrm{z}}\right)+\rho \mathrm{g}_{\mathrm{z}}
\end{aligned}
$$

Considerou-se o modelo Herschel-Bulkley para a representação reológica do fluido nãoNewtoniano, Equação 5. 


$$
\tau=\tau_{0}+K(\dot{\gamma})^{n}
$$

\subsection{Geração das Geometrias e Malhas}

Foi utilizado o software GAMBIT $^{\circledR}$ para a construção das geometrias dos anulares, que possuíam as seguintes dimensões: diâmetro externo de $100 \mathrm{~mm}$, diâmetro interno de $50 \mathrm{~mm}$, comprimento de $0,5 \mathrm{~m}$, altura de obstrução de 0,6 e $12 \mathrm{~mm}$, considerando-se o desvio ou não do cilindro interno para os casos concêntricos $(E=0)$ e excêntricos $(E=0,23)$, conforme mostra a Figura 1. Utilizou-se células hexaédricas na geração das malhas estruturadas para as geometrias. As malhas foram selecionadas após um estudo sistemático de refinamento usando quatro diferentes malhas: 8x76x123, 10x96x153, 11x108x174, 12x118x194 (direções radial, tangencial e axial) para as malhas concêntricas; e

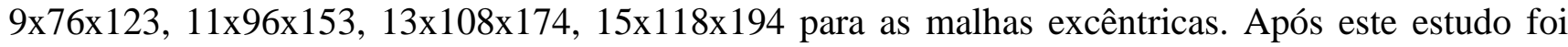
selecionado o refinamento de $11 \times 108 \times 174$ para os anulares concêntricos e $13 \times 108 \times 174$ para os anulares excêntricos. O número total de células foi de 206712 ( $\mathrm{E}=0)$ e 244296 células $(\mathrm{E}=0,23)$.

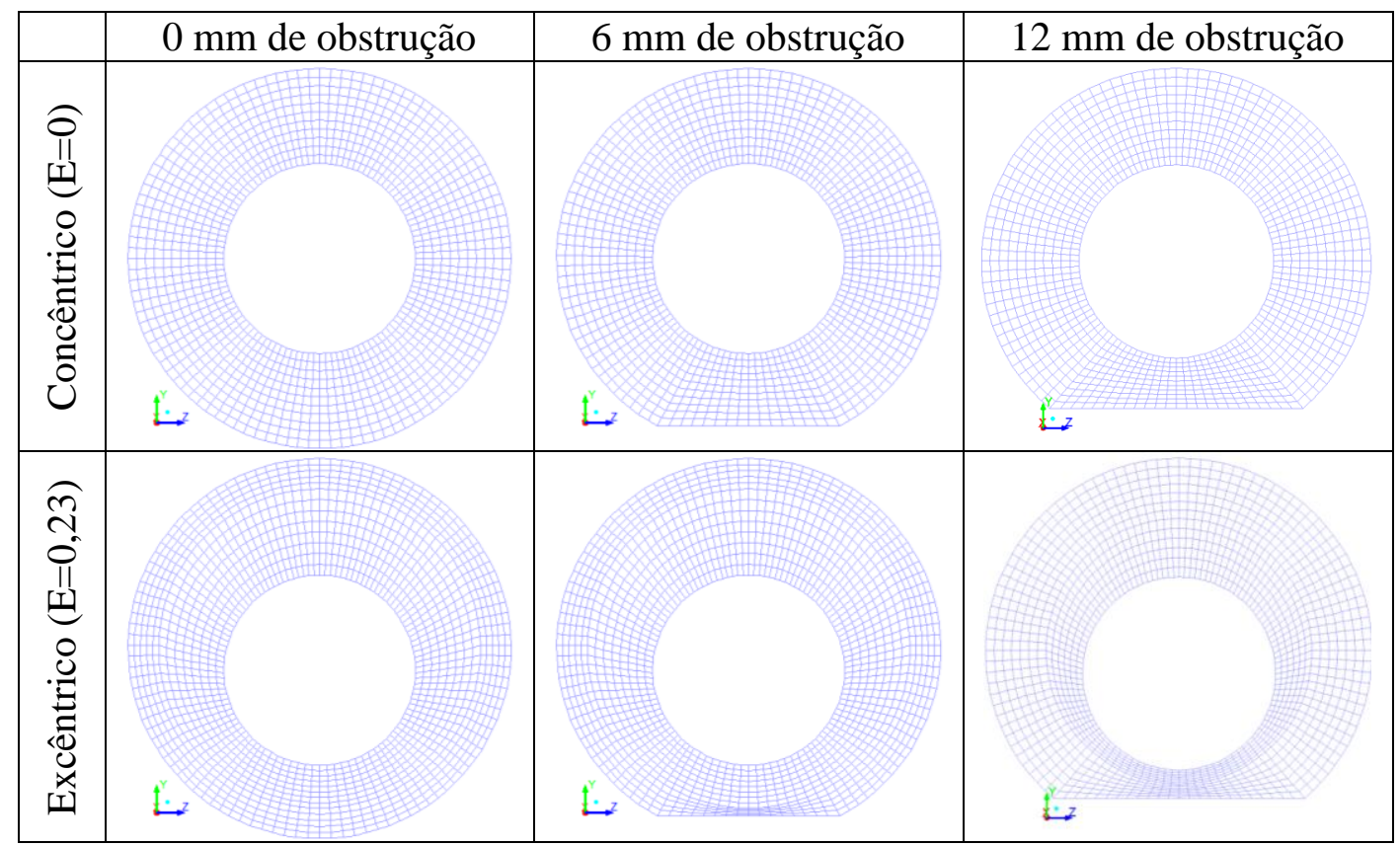

Figura 1 - Malhas geradas para os anulares de 0,5 m com obstruções de 0,6 e $12 \mathrm{~mm}$.

Uma observação muito importante a se fazer sobre as malhas foi a criação de interfaces nas malhas excêntricas. Estas interfaces consistem em regiões limítrofes que separam duas zonas de células adjacentes, e sua criação serve para permitir que estas zonas de células adjacentes se movam com velocidades diferentes. Desta forma, foi possível utilizar a condição de malha móvel para um volume de células enquanto que para o outro volume foi definida a condição de malha fixa, ao mesmo tempo. As células que pertencem a interface móvel deslizam sobre as células que pertencem a interface fixa, e esta condição é chamada de malhas deslizantes. Isto foi necessário para produzir uma movimentação excêntrica do cilindro interno, que é estudada neste trabalho, Figura 2. 


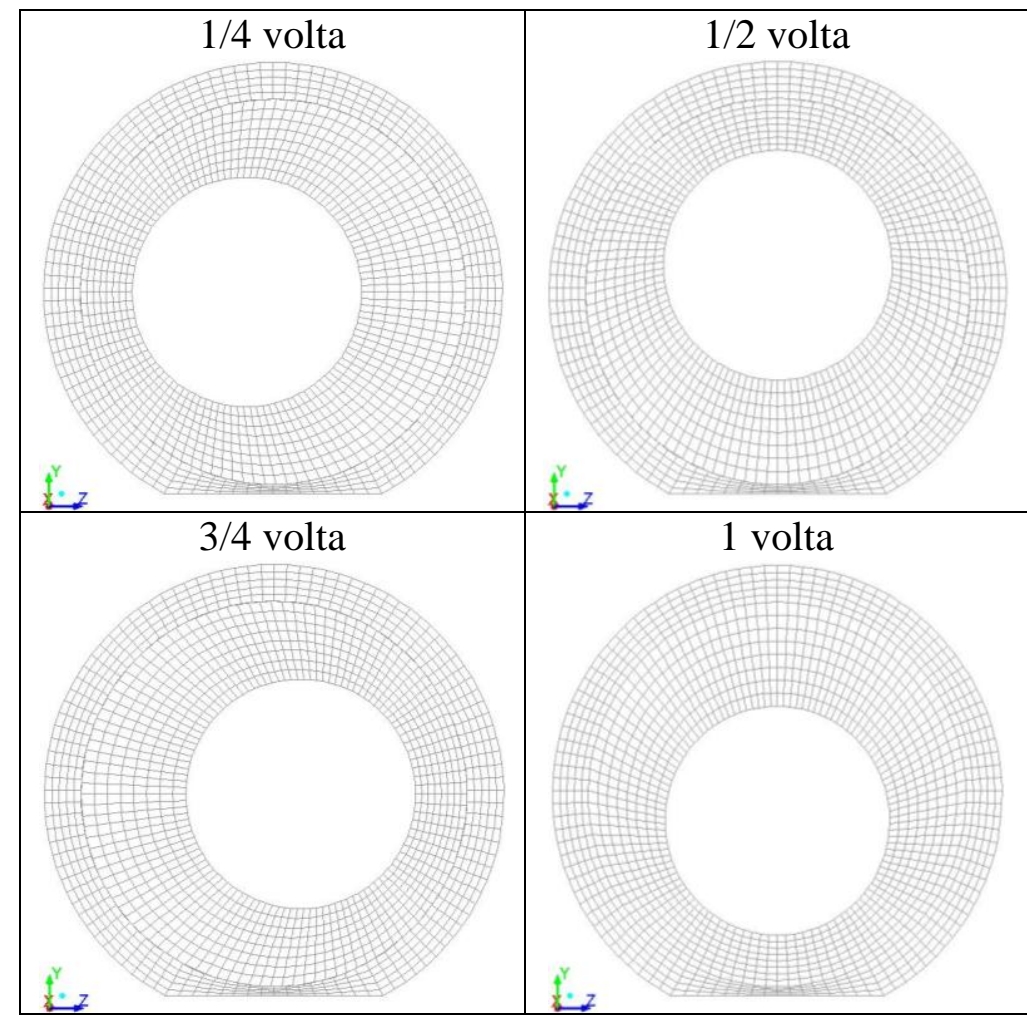

Figura 2 - Ilustração da movimentação excêntrica do cilindro interno no anular E=0,23 e 6 mm.

\subsection{Condições das Simulações}

As simulações foram conduzidas no software FLUENT $^{\circledR}$ 14.0, que acompanha o pacote ANSYS $^{\circledR}$ 14.0, sendo realizadas em regime laminar, utilizando condições de contorno periódicas na direção axial. Foram utilizadas vazões de $3 \mathrm{~m}^{3} / \mathrm{h}$ e $9 \mathrm{~m}^{3} / \mathrm{h}$ para fluidos não-Newtonianos do tipo Herschel-Bulkley com parâmetros do modelo correspondentes a um fluido de $0,1 \%$ de GX $\left(\tau_{0}=0,54\right.$ $\left.\mathrm{Pa}, K=0,0087 \mathrm{~Pa}^{n}{ }^{n}, n=0,94\right)$ e a um outro fluido com 0,5\% de GX ( $\tau_{0}=2,29 \mathrm{~Pa}, K=0,6461$ Pa.s $\left.{ }^{n}, n=0,43\right)$. Foi também estudado o efeito da rotação do cilindro interno sobre o escoamento $(0 \mathrm{e}$ $400 \mathrm{rpm}$ ). Para os casos concêntricos, a rotação era definida como uma condição de contorno na parede do tubo interno e os casos eram simulados em regime permanente. Para os casos excêntricos, a simulação era conduzida em regime permanente para os casos sem rotação, e em regime transiente para os casos em que o cilindro interno deveria rotacionar. Nestes casos era habilitada a condição de malha móvel (moving mesh) para o volume de células central que deveria rotacionar em torno do eixo $x$, o que levava a uma rotação excêntrica do cilindro interno no anular, como mostrado na Figura 2. Foi utilizado o algoritmo SIMPLE para o acoplamento pressão-velocidade e o esquema PRESTO! para a discretização da pressão. Como estratégia de discretização das componentes da equação do movimento optou-se pela escolha de esquemas de interpolação do tipo UPWIND de primeira ordem. O critério de convergência adotado para os resíduos da equação da continuidade e as componentes da equação do movimento foi de no máximo $1 \times 10^{-5}$. Para os casos excêntricos foi definida a interface que separa as duas zonas de células computacionais. As simulações numéricas foram realizadas em 
quatro supercomputadores equipados com processadores Xeon (24 cores) com 32 GB de RAM cada. Os casos concêntricos eram simulados por cerca de 30 minutos cada, enquanto que os casos excêntricos levavam cerca de 10 dias de simulação cada.

\subsection{Validação do Modelo}

Para validar os modelos e métodos utilizados neste trabalho foi feita a comparação da previsão da queda de pressão e perfis de velocidade em anulares simulados com o FLUENT $^{\circledR} 14.0$ usando dados da literatura. A Figura 3 (a) apresenta os resultados simulados e os obtidos em um estudo experimental de FORDHAM et al., (1991) para um anular concêntrico ( $d_{0}=5 \mathrm{~cm} \mathrm{e} d_{i}=4 \mathrm{~cm}$ ) usando um fluido não-Newtoniano do tipo Herschel-Bulkley ( $\left.\tau_{0}=1,59 \mathrm{~Pa}, K=0,143 \mathrm{~Pa} \cdot \mathrm{s}^{n}, n=0,54\right)$. A Figura 3 (b) mostra a comparação entre os perfis de velocidade axial simulados e os obtidos no estudo experimental de NOUAR et al., (1998) em um anular concêntrico ( $d_{0}=65 \mathrm{~mm}$ e $d_{i}=40 \mathrm{~mm}$ ) usando um fluido não-Newtoniano ( $\tau_{0}=26,54 \mathrm{~Pa}, K=20,93 \mathrm{~Pa} \cdot \mathrm{s}^{n}, n=0,35$ ) sem rotação do cilindro interno e um outro fluido ( $\tau_{0}=32 \mathrm{~Pa}, K=12,09 \mathrm{~Pa} \cdot \mathrm{s}^{n}, n=0,43$ ) para caso com rotação.

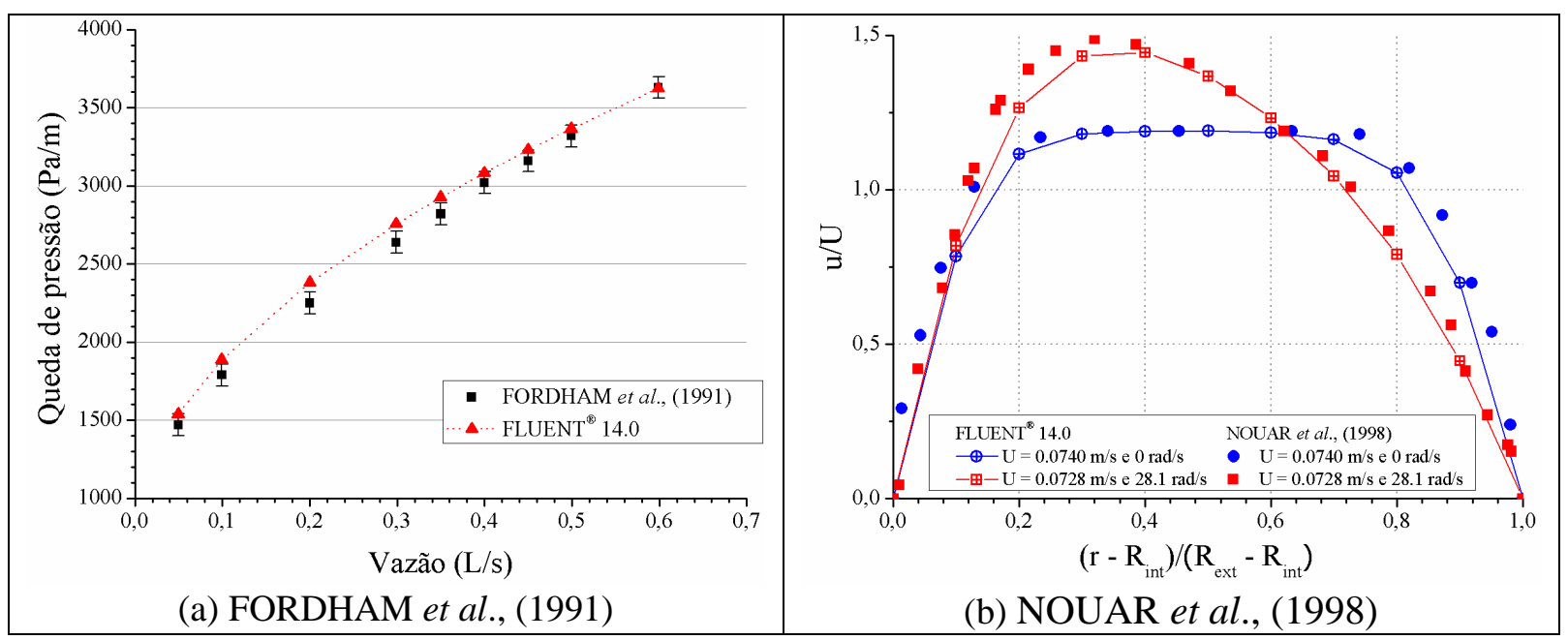

Figura 3 - Comparação de resultados simulados pelo FLUENT $^{\circledR} 14.0$ com a literatura.

\section{RESULTADOS E DISCUSSÕES}

As Figuras 4 e 5 apresentam os perfis de distribuição radial de velocidade axial em (m/s) para os anulares com diferentes alturas de obstrução concêntricos e com excentricidade de 0,23, respectivamente. Os perfis foram obtidos em uma linha na direção y na seção anular após o alcance do regime permanente ou após 8 voltas para os casos simulados em regime transiente. Em cada figura é possível se observar o efeito da vazão de fluido e da rotação do cilindro interno. A comparação com a figura abaixo/acima permite observar o efeito da concentração de goma Xantana, e a comparação com a figura à direita/esquerda permite observar o efeito da altura de obstrução sobre o escoamento. Na Figura 5 as linhas pontilhadas na vertical somente indicam a posição da interface gerada nas malhas excêntricas, permitindo visualizar que ela não afetou a qualidade dos resultados obtidos. 


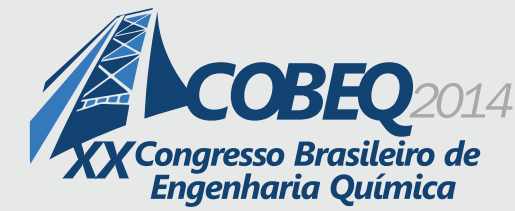

19 a 22 de outubro de 2014

Florianópolis/SC
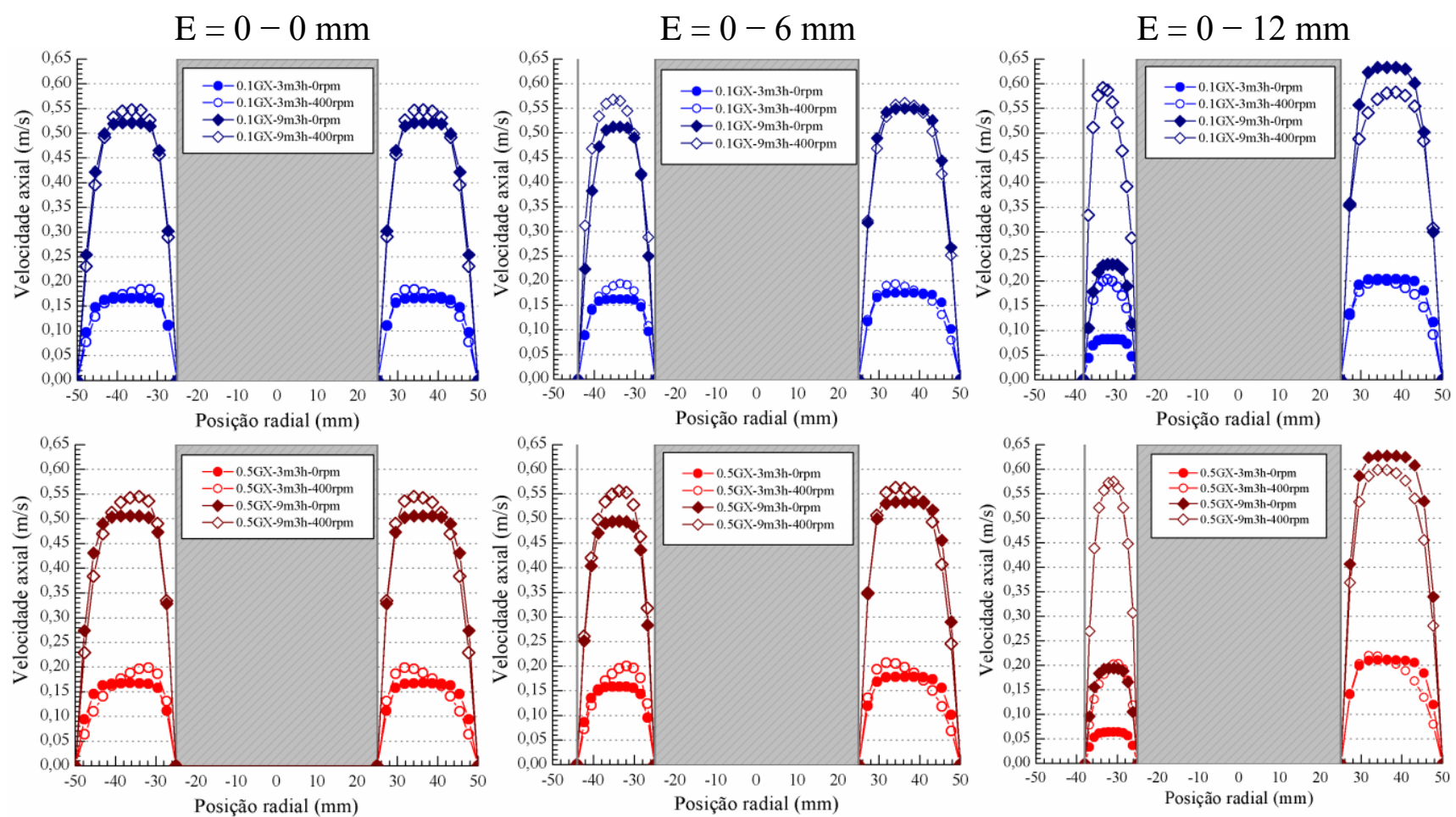

Figura 4 - Perfil de distribuição de velocidade axial (m/s) para os anulares concêntricos.
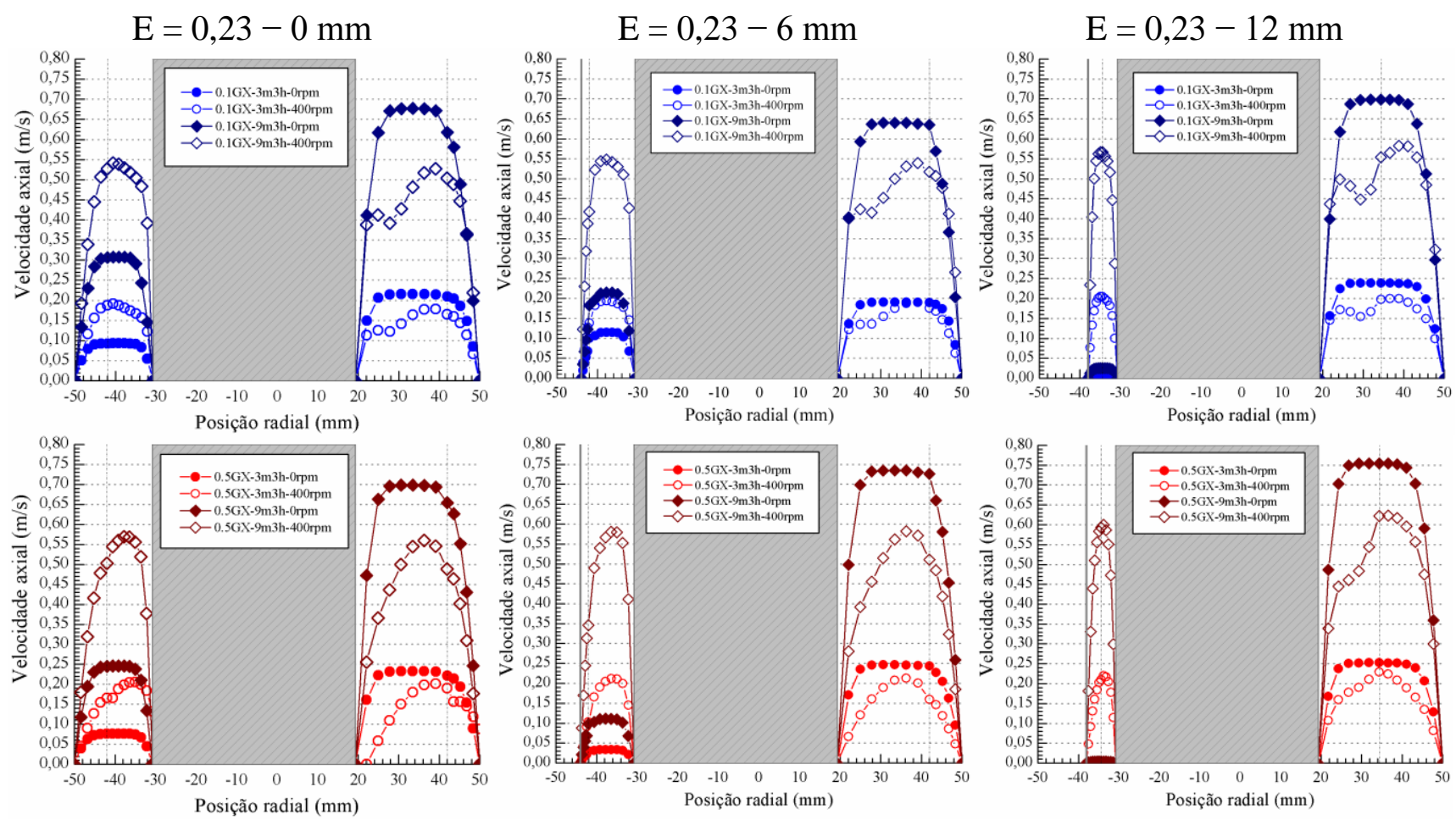

Figura 5 - Perfil de distribuição de velocidade axial $(\mathrm{m} / \mathrm{s})$ para os anulares excêntricos $(\mathrm{E}=0,23)$. 


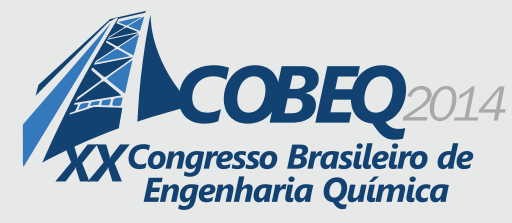

19 a 22 de outubro de 2014
Florianópolis/SC

A Figura 4 mostrou que são obtidos perfis de velocidade axiais simétricos nos dois lados opostos dos anulares concêntricos sem obstrução, como era esperado para esta configuração. No entanto, com a presença de obstrução de $6 \mathrm{~mm}$ já é possível se observar um pequeno desvio deste comportamento para vazões mais elevadas, sendo que este desvio é bastante acentuado na presença de obstrução de $12 \mathrm{~mm}$, situação em que o perfil de velocidade já se mostra preferencialmente na região da seção anular de maior gap (distância entre os cilindros externo e interno). Em todos os casos notase que a introdução da rotação do cilindro interno anula completamente essa assimetria nos perfis de velocidades axiais geradas pela presença da obstrução. Já na Figura 5, para os casos dos anulares com excentricidade de 0,23 sem rotação, observou-se que as velocidades axiais obtidas no lado de menor gap do anular foram sempre menores que do lado de maior gap, sendo esta diferença acentuada com o aumento da altura da obstrução no anular. Assim, a excentricidade introduziu uma assimetria nos perfis de velocidade axial. Essa assimetria foi praticamente eliminada com a rotação do cilindro interno que fez com fossem alcançadas velocidades similares em ambos os lados do anular, diminuindo ou eliminando a tendência de canalização da velocidade em uma região e estagnação em outra, como pode ser observado nos casos com rotação da Figura 5. Para alguns casos de E=0,23 usando fluido com $0,1 \%$ GX são observados dois picos de velocidade máxima, situação esta que já foi reportada na literatura, como por exemplo, no trabalho de ESCUDIER et al. (2000). O efeito da vazão foi comum para todos os anulares, sendo que o seu incremento favorecia a obtenção de maiores velocidades axiais. O efeito do aumento da concentração de $\mathrm{GX}$, ou acentuação do caráter nãoNewtoniano do fluido, não foi evidente nestes perfis. Os valores dos gradientes de pressão correspondentes a cada um dos 48 casos estudados podem ser visualizados na Figura 6.
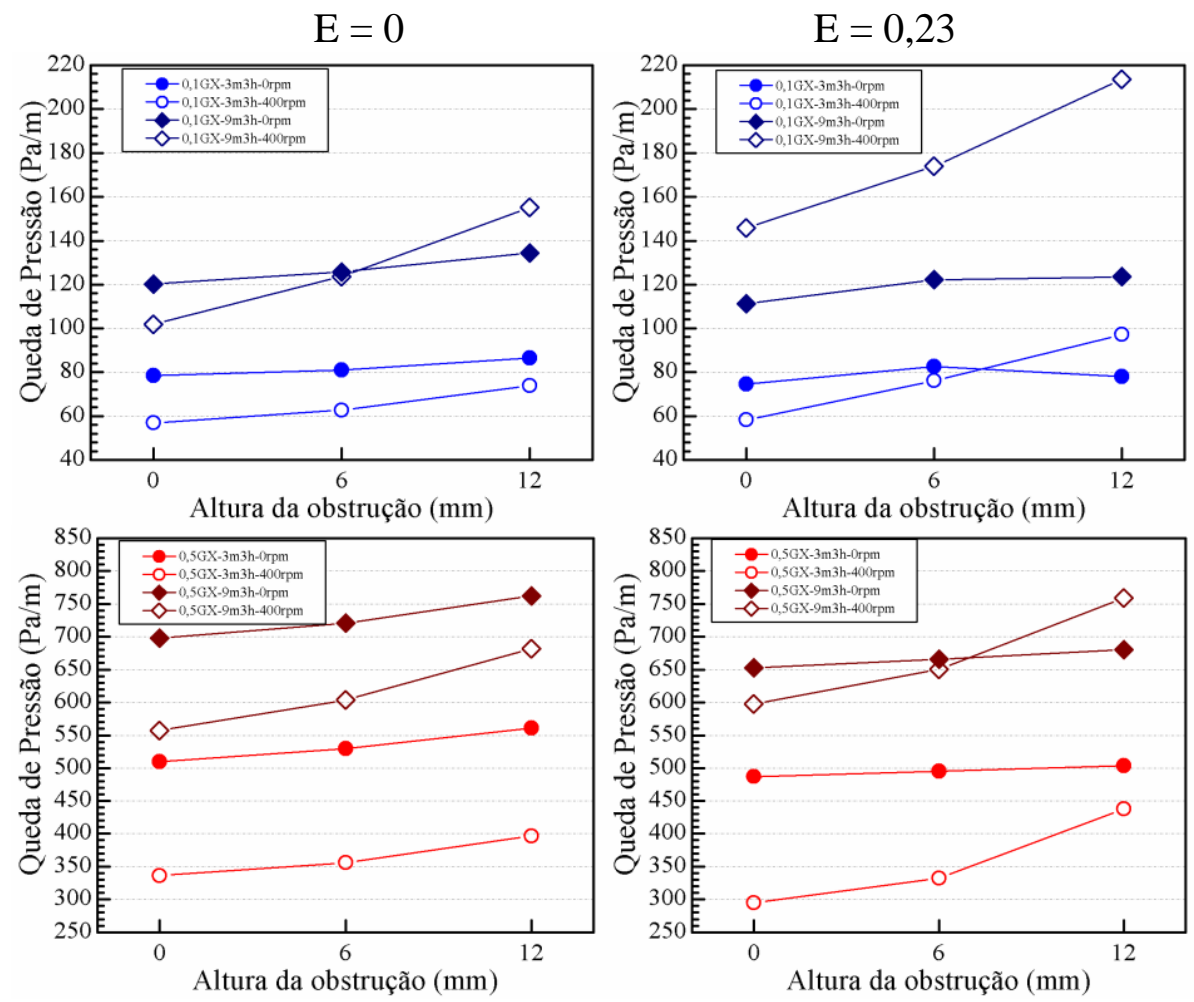

Figura 6 - Resultados de queda de pressão versus altura de obstrução. 


\section{9 a 22 de outubro de 2014 \\ Florianópolis/SC}

Observa-se na Figura 6 que a concentração de goma foi a variável que provocou maiores variações na queda de pressão, sendo que quanto maior sua concentração mais elevados eram os gradientes de pressão obtidos. O aumento da vazão ou uso de maiores obstruções também contribuíram para o acréscimo da queda de pressão nos anulares. O efeito da rotação, na maioria dos casos, foi contribuir para uma redução da queda de pressão.

\section{CONCLUSÕES}

O incremento da vazão de fluido no anular favoreceu a obtenção de maiores velocidades axiais. A excentricidade e a presença da obstrução introduziram uma assimetria nos perfis de velocidade. A rotação do eixo interno contribuiu para aumentar os valores de velocidade axial nas regiões de menor gap, auxiliando na distribuição do fluxo na região anular, o que é favorável em processos de escoamento de fluidos de perfuração em poços horizontais, já que a estagnação do fluxo provoca o surgimento de regiões de acúmulo de sólidos e aumento da queda de pressão. As quedas de pressão nos anulares eram aumentadas com o uso de fluido com maior concentração de GX e maiores vazões. A presença da obstrução elevou a queda de pressão e a introdução da rotação teve um efeito contrário. O solver comercial FLUENT $^{\circledR}$ apresentou bom desempenho para descrever o comportamento fluidodinâmico de um anular parcialmente obstruído e sua aplicação deve ser expandida no sentido de prever também o comportamento dos cascalhos no interior do espaço anular.

\section{AGRADECIMENTOS}

Agradecemos à CAPES e a PETROBRAS pelo apoio financeiro, e a Fundação de Amparo à Pesquisa do Estado de Minas Gerais (FAPEMIG) pelos recursos concedidos no Projeto de Participação Coletiva em Eventos Técnicos-Científicos (PCE-00082-14).

\section{REFERÊNCIAS}

ESCUDIER, M.P., GOULDSON, I.W., OLIVEIRA, P.J., PINHO, F.T., Effects of inner cylinder rotation on laminar flow of a Newtonian fluid through an eccentric annulus. Int. J. Heat Fluid Flow v.21, p. 92-103, 2000.

ESCUDIER, M.P., OLIVEIRA, P.J., PINHO, F.T., Fully developed laminar flow of purely viscous non-Newtonian liquids through annuli, including the effects of eccentricity and inner-cylinder rotation. Int. J. Heat and Fluid Flow, v. 23, p. 52-73, 2002.

FORDHAM E.J., BITTLESTON S.H., TEHRANI M.A., Viscoplastic flow in concentric annuli, pipes and slots, Ind. Eng. Chem. Res., v. 30, p. 517-524, 1991.

LOUREIRO, B.V., Escoamento Secundário em um Anular Parcialmente Obstruído com Rotação do Cilindro Interno, PUC, Rio de Janeiro - RJ (tese de doutorado) 181 p., 2004.

NOUAR, C., DESAUBRY, C., ZENAIDI, H., Numerical and experimental investigation of thermal convection for a thermodependent Herschel-Bulkley fluid in an annular duct with rotating inner cylinder. Eur. J. Mech. B., v. 17, p. 875-900, 1998.

PEREIRA, F.A.R., Escoamento Laminar de Líquidos Não-Newtonianos em Seções Anulares: Estudos de CFD e Abordagem Experimental, UFU, Uberlândia - MG (tese de doutorado) 229 p., 2006.

THOMAS, J.E., Fundamentos de Engenharia de Petróleo, 2.ed., RJ, Ed. Interciência, 271 p., 2001. 